\title{
COMPARISON OF ALGORITHMS FOR ESTIMATING ULTRASOUND ATTENUATION WHEN PREDICTING CERVICAL REMODELING IN A RAT MODEL
}

\author{
Timothy A. Bigelow ${ }^{1}$, Yassin Labyed ${ }^{1,2}$, Barbra L. McFarlin ${ }^{3}$,Ellora Sen-Gupta ${ }^{4}$, William D. O'Brien, \\ $J{ }^{4}$ \\ ${ }^{1}$ Department of Electrical and Computer Engineering, Iowa State University, Ames, IA \\ ${ }^{2}$ Los Alamos National Laboratory, Los Alamos, NM \\ ${ }^{3}$ Department of Women Children and Family Health Science, University of Illinois, Chicago, IL \\ ${ }^{4}$ Bioacoustics Research Laboratory, Department of Electrical and Computer Engineering, University of \\ Illinois, Urbana, IL
}

\begin{abstract}
The future of biomedical imaging involves obtaining quantitative diagnostic information about tissue structure and function rather than just relying on qualitative structural assessments. Ultrasound attenuation or loss of signal within tissue can be used to quantify micro-structural changes in the tissue consistent with cervical remodeling. In order to obtain the best estimates, the algorithms used to estimate ultrasound attenuation (spectral log difference method, spectral difference method, and hybrid method) need to be optimized and compared. In this study, the effects of the inhomogeneities within the ROI on the accuracy of the 3 algorithms were studied, and the optimal ROI size (number of independent echoes laterally and number of pulse lengths axially) was quantified for each method. The conclusions were then validated by analyzing an ultrasound image of a pregnant rat cervix from a custom-made high-frequency ultrasound imaging system.
\end{abstract}

Index Terms - ultrasound imaging, tissue characterization

\section{INTRODUCTION}

The ultrasonic attenuation coefficient is an important parameter to characterize tissue pathologies and has shown potential for assessing the risk of premature delivery by predicting cervical remodeling [1-3]. Cervical remodeling precedes the contractions of preterm labor and birth. Our research seeks to determine whether ultrasonic attenuation can be used as a noninvasive early biomarker of impending preterm labor and birth. The spectral difference method, the spectral log difference method, and the hybrid method have all been used for estimating the attenuation in human tissue previously [1, 4-10]. The spectral difference method uses the decrease of the different frequency components of the power spectrum with respect to depth to estimate the attenuation coefficient [9]. The spectral log difference method finds the attenuation by calculating the slope of the straight line that fits the log ratio (difference between log spectra) of the two power spectra from the proximal and the distal segments of the region of interest (ROI) $[4,5,8]$. The hybrid method estimates the attenuation coefficient slope by measuring the downshift in the center frequency of the spectra with depth after multiplying by a Gaussian filter [10]. The accuracy and the precision of these methods are strongly dependent on the ROI size (the number of independent echoes laterally and the number of pulse lengths axially) and on the level of homogeneity within the ROI. However, there has been no quantitative comparison of the minimum ROI size that is required for each method to obtain certain accuracy and precision in the attenuation coefficient estimates. In this study, we use simulations, tissue mimicking phantom experiments, and ultrasound scans of a rat cervix in vivo to determine the optimal ROI sizes for each algorithm and the dependence of the algorithm on tissue inhomogeneities.

\section{PROCEDURES}

\subsection{Computer simulation procedures}

Computer simulations were used to obtain 3 different data sets of RF backscattered signals using a Gaussian focused beam ( $5 \mathrm{~cm}$ focal length, $10 \mathrm{MHz}$ center frequency, $0.5 \mu$ s pulse duration corresponding to $\sim 0.4 \mathrm{~mm}$ spatial pulse length, and a 50\% $-3 \mathrm{~dB}$ bandwidth). The first data set was used as a reference to correct for the diffraction properties of the source and consisted of $10 \mu \mathrm{m}$ radius spherical shell scatterers similar to a glass bead phantom that would be used in the experiments. The remaining two data sets simulated biological tissue and had randomly distributed $20 \mu \mathrm{m}$ effective radius scatterers with a Gaussian correlation function. The attenuation coefficients of the samples and the reference were 0.7 and $0.5 \mathrm{~dB} \mathrm{~cm}^{-1} \mathrm{MHz}^{-1}$, respectively. The number density of scattering structures 
for the simulated cases are given in Table 1. After generating the backscattered waveforms, the three different algorithms were applied to determine the attenuation of the different regions for ROI's of varying pulse durations (axial dimension) and number of independent echoes (lateral dimension).

Table 1: Summary of scattering properties.

\begin{tabular}{|c|c|c|c|}
\hline & Reference & Sample 1 & Sample 2 \\
\hline $\begin{array}{c}\text { \# Density } \\
\text { Above Focal } \\
\text { Plane }\end{array}$ & $100 \mathrm{~mm}^{-3}$ & $100 \mathrm{~mm}^{-3}$ & $200 \mathrm{~mm}^{-3}$ \\
\hline $\begin{array}{c}\text { \# Density } \\
\text { Below Focal } \\
\text { Plane }\end{array}$ & $100 \mathrm{~mm}^{-3}$ & $100 \mathrm{~mm}^{-3}$ & $100 \mathrm{~mm}^{-3}$ \\
\hline
\end{tabular}

\subsection{Tissue-mimicking phantom experiment procedures}

A clinical array transducer $(\sim 5.5 \mathrm{MHz}, \mathrm{L} 8-3$ Linear Array Transducer) driven by a clinical ultrasound system (z.one Ultrasound System, ZONARE Medical Systems, Inc.) was used to obtain $10 \mathrm{RF}$ data sets from a homogeneous region in a $0.7 \mathrm{~dB} \mathrm{~cm}^{-1} \mathrm{MHz}^{-1}$ tissue mimicking phantom (Gammex 406 LE 0.7, Gammex, Inc., Middleton, WI) and $1 \mathrm{RF}$ data set from a homogeneous region in a $0.5 \mathrm{~dB} \mathrm{~cm}^{-1} \mathrm{MHz}^{-1}$ tissue mimicking phantom (Gammex 406 LE 0.5, Gammex, Inc., Middleton, WI). Each set had 250 echo lines. To determine the number of uncorrelated echo lines in each ROI, the correlation coefficient was determined and every fourth echo line was determined to be uncorrelated. Thus, when determining the optimal ROI size for the phantom experiments, only every fourth echo was included. Also, using a hydrophone, we measured the pulse duration of the transducer to be approximately $0.8 \mu \mathrm{s}$ which corresponds to $\sim 0.6 \mathrm{~mm}$. After obtaining the ultrasound images, the 3 different algorithms were once again applied to determine the optimal dimensions of the ROI.

\subsection{Endovaginal scan of rat cervix procedures}

A custom scanning system was constructed consisting of a $40 \mathrm{MHz}$ spherically focused transducer with a focal length of $4.6 \mathrm{~mm}$ and a diameter of $3 \mathrm{~mm}$. The pulse duration as measured from reflecting off of a Plexiglas plane placed at the focal plane was $0.09 \mu \mathrm{s}(\sim 0.07 \mathrm{~mm})$. The transducer could be used to form a B-mode ultrasound image by operating in pulse/echo mode while being mechanically scanned with a computer controlled positioning system. The scanning system was utilized to obtain an endovaginal ultrasound image and RF echoes of a pregnant rat cervix on gestational day 21 (late gestation) as shown in Figure 1.

In addition, echoes were also obtained from a tissue mimicking phantom with known attenuation and scattering properties. The scan of the rat cervix consisted of 1001 echo lines. However, only every $10^{\text {th }}$ line was statistically independent as determined by calculating the correlation coefficient of the echoes from the reference phantom. Therefore when determining the optimal ROI size for the phantom experiments, the echoes were grouped into ten sets. For each set, only every $10^{\text {th }}$ echo was included but the starting echo was different. The attenuation estimates from each set were then averaged even though the sets were not statistically independent.

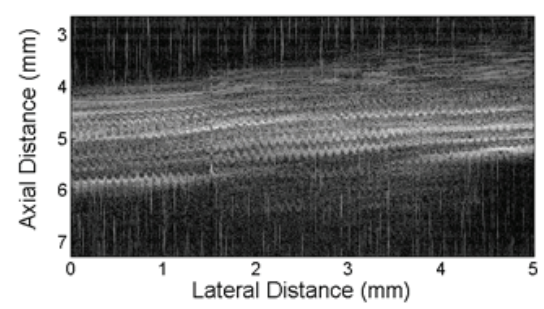

Figure 1: B-mode image of rat cervix at gestational age of 21 days.

\section{RESULTS}

\subsection{Computer simulations results}

Figure 2 shows the standard deviation of the percent error in the attenuation estimates that was obtained using the spectral difference method (Fig. 2a), the spectral log difference method (Fig. 2b), and the hybrid method (Fig. $2 \mathrm{c}$ ), versus the number of pulse lengths and the number of independent echoes per ROI when the simulated tissue was homogeneous.

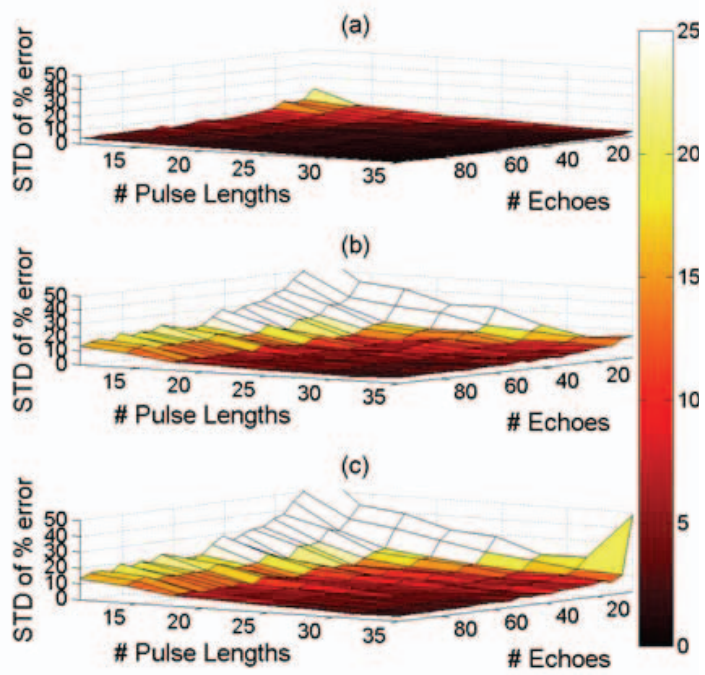

Figure 2: The standard deviation of the percent error in the attenuation estimates as a function of ROI size for the (a) spectral difference algorithm, (b) the spectral log difference method, and (c) the hybrid method when the ROI is homogeneous found using computer simulations. 
Based on these plots, we observed that the standard deviation of the percent error decrease with increasing ROI length axially and increasing number of echoes laterally for all the attenuation measurement techniques. The precision of the estimates is better at smaller ROI's when using the spectral difference method and comparable when using the spectral log difference method or the hybrid method.

The results for changes in scatterer number density with depth are summarized in Figure 3. In this figure, the standard deviation of the percent error versus ROI size is shown the spectral log difference method (Fig. 3a) and the hybrid method (Fig. 3b). The precision found using the spectral difference method is not shown because there was greater than a $100 \%$ bias in these estimates due to the change in backscatter as a function of depth. A bias was not observed for the spectral log difference method or the hybrid method. Once again, the precision was comparable when using the spectral log difference method or the hybrid method.

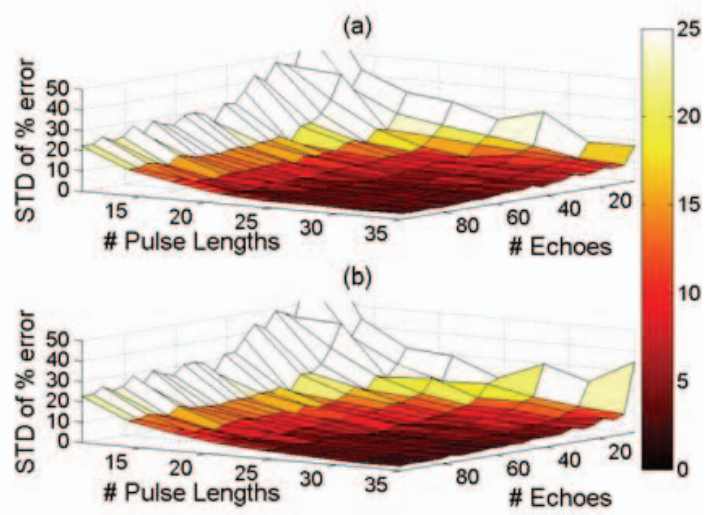

Figure 3: The standard deviation of the percent error in the attenuation estimates as a function of ROI size for the (a)

the spectral log difference method and (b) the hybrid method when the number density of scatterers varies in the

ROI found using computer simulations.

\subsection{Tissue-mimicking phantom experiment results}

Figure 4 shows the precision of the three different algorithms when using a homogeneous tissue mimicking phantom. Once again, the precision of the estimates was better for smaller ROI's when using the spectral difference method and is comparable for the log difference and the hybrid method. The precision of all of the algorithms improved as the size of the ROI increased.

\subsection{Endovaginal scan of rat cervix results}

When analyzing the scans from the rat cervix, we only applied the spectral difference algorithm and the spectral log difference algorithm. The hybrid method was not tested because our earlier results indicated that its performance was not significantly different from the log difference method, but the hybrid method is more computationally intensive. The spectral difference algorithm yielded a negative attenuation for all of the ROI sizes tested. A negative attenuation is not a physically reasonable quantity. These inaccurate estimates when using the spectral difference method likely result from inhomogeneities within the ROI. Namely, there was an increase in backscatter with increasing depth into the tissue that is also visible on the Bmode image.

(a)

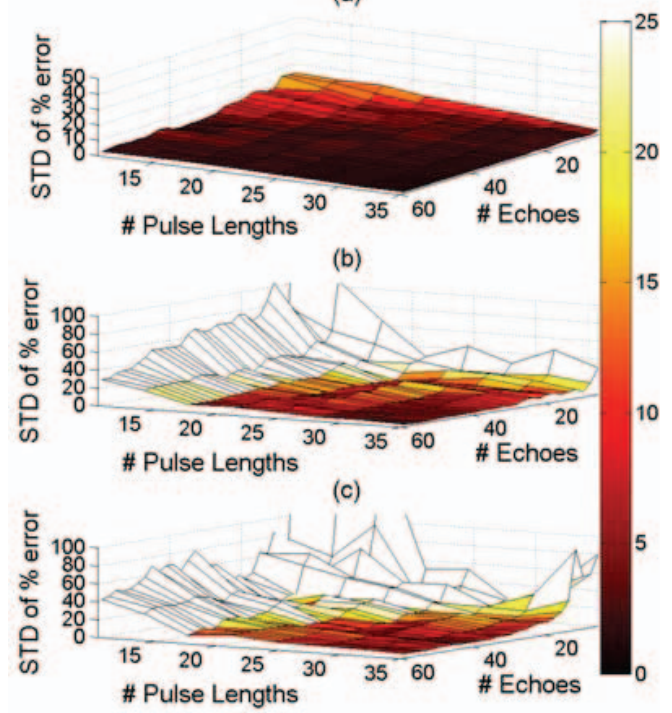

Figure 4: The standard deviation of the percent error in the attenuation estimates as a function of ROI size for the (a) spectral difference algorithm, (b) the spectral log difference method, and (c) the hybrid method when the ROI is homogeneous found using tissue mimicking phantom experiments.

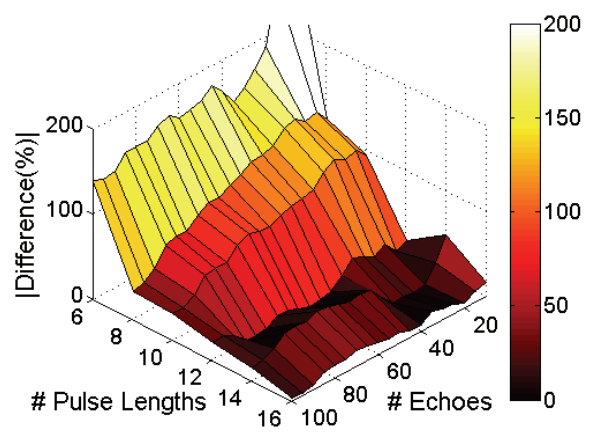

Figure 5: The percent difference in the attenuation estimate when using the spectral log difference algorithm between the largest ROI size allowed by the tissue sample and the current ROI size as a function of the ROI size.

When analyzing the results for the log difference algorithm, it was not possible to calculate the standard deviation for each ROI size. This is because the true attenuation of the cervix was not known a priori and may 
vary from animal to animal. Therefore, instead we calculated the percent difference between the estimate at the current ROI size and the estimate at the largest ROI size. This percent difference is shown in Figure 5.

\section{DISCUSSION/CONCLUSION}

In this study, we compared the precision of the 3 algorithms for estimating the attenuation; the spectral difference algorithm, the spectral log difference algorithms, and the hybrid algorithm. When the tissue was homogenous, the spectral difference algorithm resulted in the best precision especially at smaller ROI sizes. However, when the tissue was not homogeneous, the spectral difference algorithm gave biased estimates. The other two algorithms had comparable precision, were less sensitive to tissue inhomogeneities, but required larger ROI sizes.

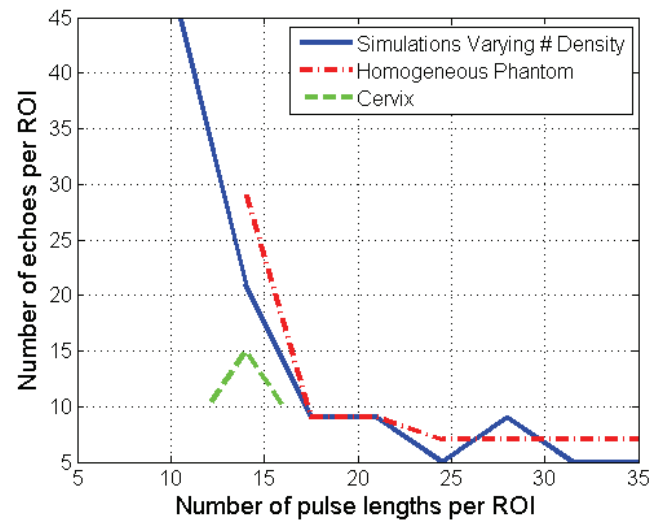

Figure 6: The number of echoes required to have a standard deviation of less than $25 \%$ (simulations and homogeneous phantom) or a percent difference in the attenuation estimate of less than $25 \%$ (cervix) as a function of the number of pulse lengths in the ROI.

In order to better facilitate a comparison between the simulations, phantom experiments, and rat cervix scan, the number of echoes required to obtain a standard deviation or percent difference of $25 \%$ was found for each ROI axial size in pulse lengths. If the variance or percent difference was never less than $25 \%$ for a ROI size, then that axial size was skipped in the analysis. The results of this comparison are shown in Figure 6. From this analysis, it is clear that when implementing the spectral log difference algorithm, it is critical to have a sufficient number of pulse lengths per ROI. For a variance on the order of $25 \%$, the ROI should be at least 12 to 20 pulse lengths in the axial direction. The number of independent echoes is not nearly as critical with 10 to 20 independent echoes being sufficient provided the ROI is 15 to 20 pulse lengths deep. Using ROI's of this size should allow us to quantify the attenuation of the rat cervix in future animal studies.

\section{ACKNOWLEDGEMENTS}

This project was supported by a subcontract with the University of Illinois-Chicago made possible by Grant \# R21HD058705-01A2 from the National Institutes of Health, by NIH Grant R01CA111289, by Iowa State University, and by grant \# KL2-RR029877 from the University of Illinois Chicago Center for Clinical and Translational Science supported by Award Number UL1RR029879 from the National Center for Research Resources. The content is solely the responsibility of the authors and does not necessarily represent the official views of the National Institutes of Health.

\section{REFERENCES}

[1] B. L. McFarlin, W. D. O'Brien, Jr., M. L. Oelze, J. F. Zachary, and R. C. White-Traut, "Quantitative Ultrasound Assessment of the Rat Cervix," J Ultrasound Med, vol. 25, pp. 1031-1040, August 1, 20062006.

[2] T. A. Bigelow, B. L. McFarlin, W. D. O'Brien Jr, and M. L. Oelze, "In vivo ultrasonic attenuation slope estimates for detecting cervical ripening in rats: Preliminary results," The Journal of the Acoustical Society of America, vol. 123, pp. 1794-1800, 2008.

[3] B. L. McFarlin, T. A. Bigelow, Y. Laybed, W. D. O'Brien, M. L. Oelze, and J. S. Abramowicz, "Ultrasonic attenuation estimation of the pregnant cervix: a preliminary report," Ultrasound in Obstetrics and Gynecology, vol. 36, pp. 218-225, 2010.

[4] R. Kuc and M. Schwartz, "Estimating the Acoustic Attenuation Coefficient Slope for Liver from Reflected Ultrasound Signals," Sonics and Ultrasonics, IEEE Transactions on, vol. 26, pp. 353361, 1979.

[5] R. Kuc, "Clinical Application of an Ultrasound Attenuation Coefficient Estimation Technique for Liver Pathology Characterization," Biomedical Engineering, IEEE Transactions on, vol. BME-27, pp. 312-319, 1980.

[6] T. J. Hall, M. F. Insana, L. A. Harrison, and G. G. Cox, "Ultrasonic measurement of glomerular diameters in normal adult humans," Ultrasound in Medicine \& Biology, vol. 22, pp. 987-997, 1996.

[7] T. A. Bigelow, B. L. McFarlin, W. D. O'Brien, Jr., and M. L. Oelze, "In vivo ultrasonic attenuation slope estimates for detecting cervical ripening in rats: Preliminary results," The Journal of the Acoustical Society of America, vol. 123, pp. 1794-1800, 2008.

[8] R. Kuc, "Estimating acoustic attenuation from reflected ultrasound signals: Comparison of spectral-shift and spectraldifference approaches," Acoustics, Speech and Signal Processing, IEEE Transactions on, vol. 32, pp. 1-6, 1984.

[9] L. X. Yao, J. A. Zagzebski, and E. L. Madsen, "Backscatter coefficient measurements using a reference phantom to extract depth-dependent instrumentation factors," Ultrasonic Imaging, vol. 12, pp. 58-70, 1990.

[10] H. Kim and T. Varghese, "Hybrid Spectral Domain Method for Attenuation Slope Estimation," Ultrasound in Medicine \& Biology, vol. 34, pp. 1808-1819, 2008. 